\title{
Gait Identification Considering Body Tilt by Walking Direction Changes
}

\author{
Yasushi Makihara*, Ryusuke Sagawa*, Yasuhiro Mukaigawa*, Tomio Echigo ${ }^{+}$, and Yasushi Yagi* \\ * Dept. of Intelligent Media, Osaka University, 8-1 Mihogaoka, Ibaraki, Osaka, JAPAN
}

* Dept. of Engineering Informatics, Osaka Electro-Communication University, 18-8 Hatsumachi, Neyagawa, Osaka, JAPAN

Received 16th June 2008; accepted 21th July 2009.

\begin{abstract}
Gait identification has recently gained attention as a method of identifying individuals at a distance. Thought most of the previous works mainly treated straight-walk sequences for simplicity, curved-walk sequences should be also treated considering situations where a person walks along a curved path or enters a building from a sidewalk. In such cases, person's body sometimes tilts by centrifugal force when walking directions change, and this body tilt considerably degrades gait silhouette and identification performance, especially for widely-used appearance-based approaches. Therefore, we propose a method of body-tilted silhouette correction based on centrifugal force estimation from walking trajectories. Then, gait identification process including gait feature extraction in the frequency domain and learning of a View Transformation Model (VTM) follows the silhouette correction. Experiments of gait identification for circular-walk sequences demonstrate the effectiveness of the proposed method.
\end{abstract}

Keywords: Gait identification, Body tilt correction, Fourier analysis, View Transformation Model

\section{Introduction}

There is a growing necessity in modern society to identify individuals in many situations, including, surveillance and access control. For personal identification, many biometrics-based authentication methods are proposed using a wide variety of cues; fingerprint, iris, and face. On the other hand, action recognition by spacetime analysis [1] and authentication from action [2] have been studied for a recent years. Particularly, authentication by gait [3] has gained considerable attention because gait promises to enable surveillance systems to ascertain identity at a distance.

Current approaches to gait identification are mainly divided into two types: model-based [4][5][6][7] and appearance-based [8][9][10][11]. For both approaches, a gait motion is generally observed the best in a frontoparallel view, hence most of the approaches assume the gait is observed in near fronto-parallel views. The assumption is, however, unreasonable in actual surveillance scenarios, and changes of viewing or walking direction induce considerable appearance changes. Yu et al. [12] discussed the effects of view angle variation on gait identification and reported a performance drop when view difference is large.

Correspondence to: <makihara@am.sanken.osaka-u.ac.jp>

ELCVIA ISSN: 1577-5097

Published by Computer Vision Center / Universitat Autònoma de Barcelona, Barcelona, Spain 
To cope with view changes, Weinland et al. [13] propose action taxonomies with multi-view observations, and Shakhnarovich et al. [14] proposed a visual hull-based gait identification method. However, the method needs multiple-view synchronized images for all subjects. Kale et al. [15] proposed a view transformation method based on perspective projection of the sagittal plane. The method does not, however, work well because of self occlusion when view difference is large and an angle between an image plane and the sagittal plane is large. Urtasun et al. [5] proposed a 3D model-based method to treat view changes, it is, however, needs substantially high-resolution input images so as to fit the model and extract model parameters accurately.

Another method [16] integrated a frequency-domain gait feature [11] and a bilinear model as View Transformation Model (VTM) [17] which transforms a few-view features to arbitrary-view features. The method successfully adapted to view changes and illustrated the effectiveness by experiments using various-view straightwalk gait sequences.

Hence, the remaining problem is gait identification from curved-walk sequences, which comes from situations where a person walks on a curved path or entering the building from a sidewalk. In such situations, the centrifugal force is induced from the gradual walking direction changes and it tilts the walking person's body. As a result, an observed gait silhouette changes and the identification performance drops.

A basic idea for silhouette correction is appearance-based rotation correction using a principal axis of the silhouette. This method does not, however, work well because the principal axis does not always coincides with the actual body axis. For example, the principal axis of a person whose foot step or arm swing motion is asymmetric inclines to left or right even if the person stands perpendicularly.

Another approach is use of rotation invariant features. The method, however, eliminate not only body tilts by centrifugal force but also those derived from individualities which are helpful for identification in fact. For example, silhouettes of walkers with unsteady steps may include some periodic rotations and it can be a useful cue to distinguish from those with steady steps.

Therefore, we propose a method of mechanics-based silhouette correction by body tilt estimation derived from the centrifugal force. First, a walking trajectory is extracted and the centrifugal force is subsequently estimated from the trajectory. Next, the body tilt angle is estimated from a balance of the centrifugal force and the gravitational force acting the walking person. Finally, the gait silhouette is corrected by rotation and scaling based on the estimated body tilt angle.

The outline of this paper is as follows. First, gait identification process is overviewed with frequency-domain feature extraction and VTM learning described in section 2. Then, gait silhouette correction is presented with body tilt estimation for curved-walk sequences in section 3. Finally, gait identification experiments for circularwalk sequences are shown in section 4 , and conclusions and future works are given in section 5.

\section{Overview of Gait Identification Process}

This section gives a brief description of gait identification process (see [16] for detail).

\subsection{Frequency-domain Feature}

First, gait silhouettes are extracted by background subtraction and are normalized into fixed-size $(20 \times 30$ pixel) silhouettes. Because the normalized silhouette sequence is regarded as 3-D data: spatial $2 \mathrm{D}(x, y)$ and temporal 1D $(t)$, it is called Gait Silhouette Volume (GSV). Second, gait period $N_{\text {gait }}$, which is assumed to be constant for each sequence, is detected by maximizing the normalized autocorrelation of the GSV for the temporal axis. Then, the subsequences $\left\{\mathbf{S}_{i}\right\}\left(i=1,2, \ldots, N_{\text {sub }}\right)$ are picked up for every $N_{\text {gait }}$ frames from a complete sequence $\mathbf{S}$. Note that the frame range of the $i$ th subsequence $\mathbf{S}_{i}$ is $\left[i N_{\text {gait }},(i+1) N_{\text {gait }}-1\right]$.

Third, a Discrete Fourier Transformation (DFT) for the temporal axis is applied for each subsequence, and 


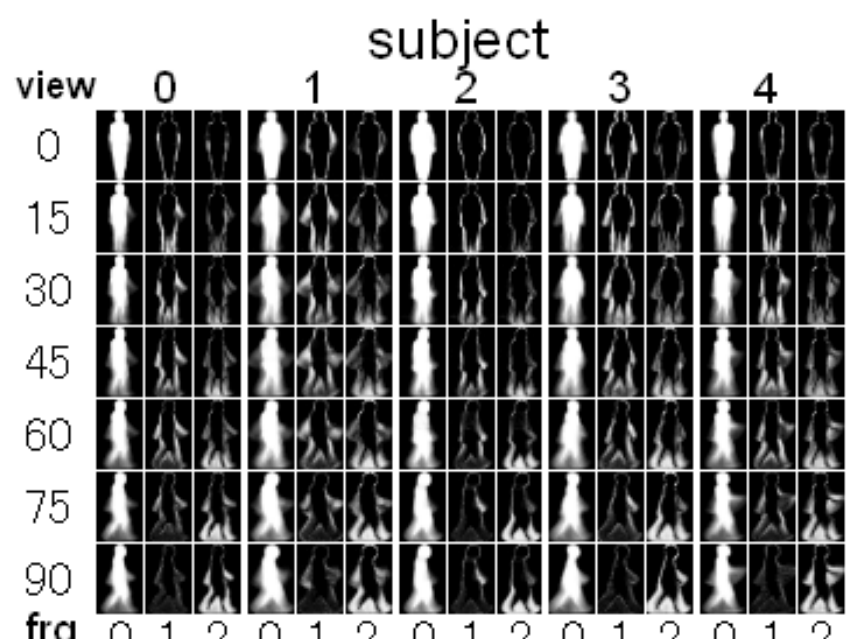

Figure 1: Gait features for multiple subjects from multiple views (every $15 \mathrm{deg}$ ). Bloc, column in each bloc, and row indicates subject, frequency, and view respectively.

amplitude spectra are calculated as

$$
\begin{aligned}
G_{i}(x, y, k) & =\sum_{n=i N_{\text {gait }}}^{(i+1) N_{\text {gait }}-1} g(x, y, n) e^{-j \omega_{0} k n} \\
A_{i}(x, y, k) & =\frac{1}{N_{\text {gait }}}\left|G_{i}(x, y, k)\right|
\end{aligned}
$$

where $\omega_{0}$ is the base angular frequency for the gait period $N_{\text {gait }}, g(x, y, n)$ is the silhouette value at position $(x, y)$ at the $n$th frame, $G_{i}(x, y, k)$ is the DFT of GSV for $k$-times the base frequency, and $A_{i}(x, y, k)$ is an amplitude spectrum for $G_{i}(x, y, k)$ normalized with the gait period $N_{\text {gait }}$. In this paper, direct-current elements $(k=0)$ (averaged silhouette) and low-frequency elements $(k=1,2)$ are chosen as experimental gait features.

Finally, a matching measure for two subsequences $d\left(\mathbf{S}_{i}, \mathbf{S}_{j}\right)$ is simply chosen as the Euclidean distance of feature vectors composed of amplitude spectra elements, and then that for two complete sequences (let probe and gallery complete sequence be $\mathbf{S}_{\mathbf{P}}$ and $\mathbf{S}_{\mathbf{G}}$ and their subsequences be $\left\{\mathbf{S}_{\mathbf{P} i}\right\}(i=1,2, \ldots)$ and $\left.\left\{\mathbf{S}_{\mathbf{G} j}\right\}(j=1,2, \ldots)\right)$ is defined as follows.

$$
D\left(\mathbf{S}_{\mathbf{P}}, \mathbf{S}_{\mathbf{G}}\right)=\operatorname{Median}_{i}\left[\min _{j}\left\{d\left(\mathbf{S}_{\mathbf{P} i}, \mathbf{S}_{\mathbf{G} j}\right)\right\}\right] .
$$

Figure 1 shows extracted amplitude spectra of multiple subjects from various directions. Note that the direction is defined as the angle formed by an optical axis and a walking direction.

\subsection{VTM}

First, the concept of the VTM is shown in Fig. 2. In the training phase, gait features are collected from a limited number of training subjects from all views to form a training set that is then used to create the VTM. Gait features from identification-target subjects which are different from the training subjects are then stored from only a few reference views. In the identification phase, this reference set is transformed into the gallery set for the probe view using the VTM and then matched.

The VTM can be seen as one of bilinear models. Lee et al. [18] used it to decompose gait dynamics and individuals, and Cuzzolin [19] exploited it to decompose gait styles, views, and individuals. Note that we 


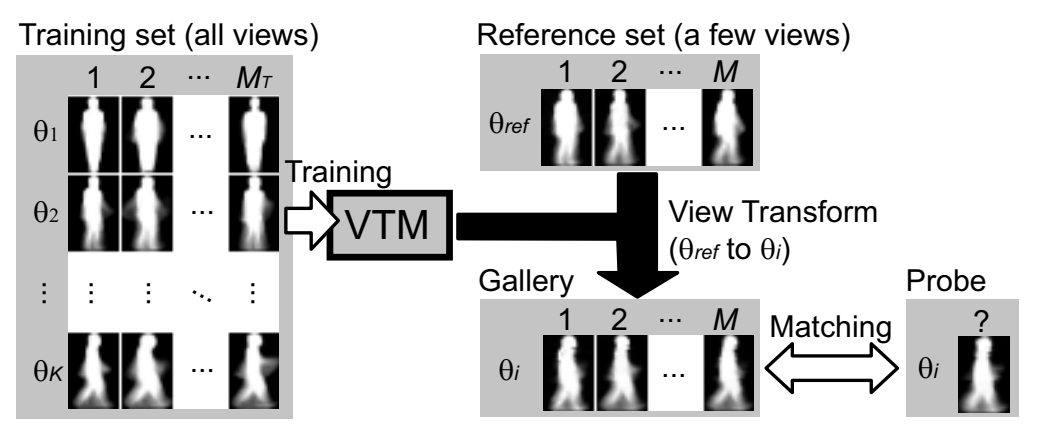

Figure 2: Problem setting of the VTM

apply the bilinear model (VTM) to decompose views and individuals in cooperation with frequency-domain gait features.

Then, the formulation of a VTM can be briefly described below. Views are first quantized into $K$ views. Let $\mathbf{a}_{\theta_{k}}^{m}$ be an $N_{A}$ dimensional feature vector for the $k$ th view of the $m$ th subject. Supposing that the feature vectors for $K$ views of $M$ subjects are obtained as a training set, a matrix can be constructed whose row indicates view changes and whose column indicates each subject; and so can be decomposed by Singular Value Decomposition (SVD) as

$$
\left[\begin{array}{ccc}
\mathbf{a}_{\theta_{1}}^{1} & \cdots & \mathbf{a}_{\theta_{1}}^{M} \\
\vdots & \ddots & \vdots \\
\mathbf{a}_{\theta_{K}}^{1} & \cdots & \mathbf{a}_{\theta_{K}}^{M}
\end{array}\right]=U S V^{T}=\left[\begin{array}{c}
P_{\theta_{1}} \\
\vdots \\
P_{\theta_{K}}
\end{array}\right]\left[\mathbf{v}^{1} \cdots \mathbf{v}^{M}\right]
$$

where $P_{\theta_{k}}$ is the $N_{A} \times M$ submatrix of $U S$, and $\mathbf{v}^{m}$ is the $M$ dimensional column vector. The vector $\mathbf{v}^{m}$ is an intrinsic feature vector of the $m$ th subject and is independent of views. The submatrix $P_{\theta_{k}}$ is a projection matrix from the intrinsic vector $\mathbf{v}$ to the feature vector for view $\theta_{k}$, and is common for all subjects. Thus, the feature vector $\mathbf{a}_{\theta_{i}}^{m}$ for the view $\theta_{i}$ of the $m$ th subject is represented as

$$
\mathbf{a}_{\theta_{i}}^{m}=P_{\theta_{i}} \mathbf{v}^{m} .
$$

Therefore, feature vectors from a few reference views $\left\{\theta_{\text {ref }}(1), \cdots, \theta_{\text {ref }}(k)\right\}$ are represented by

$$
\left[\begin{array}{c}
\mathbf{a}_{\theta_{r e f}(1)}^{m} \\
\vdots \\
\mathbf{a}_{\theta_{r e f}(k)}^{m}
\end{array}\right]=\left[\begin{array}{c}
P_{\theta_{r e f}(1)} \\
\vdots \\
P_{\theta_{r e f}(k)}
\end{array}\right] \mathbf{v}^{m}
$$

Then, the intrinsic vector $\mathbf{v}^{m}$ is estimated by least-squre method as

$$
\hat{\mathbf{v}}^{m}=\left[\begin{array}{c}
P_{\theta_{r e f}(1)} \\
\vdots \\
P_{\theta_{r e f}(k)}
\end{array}\right]^{+}\left[\begin{array}{c}
\mathbf{a}_{\theta_{r e f}(1)}^{m} \\
\vdots \\
\mathbf{a}_{\theta_{r e f}}^{m}(k)
\end{array}\right],
$$

where superscript ${ }^{+}$means pseudo inverse. Finally, feature vector transformation from a few reference views to another view $\theta_{i}$ is now easily obtained by substituting the above $\hat{\mathbf{v}}$ to eq. (5) as

$$
\hat{\mathbf{a}}_{\theta_{i}}^{m}=P_{\theta_{i}}\left[\begin{array}{c}
P_{\theta_{r e f}(1)} \\
\vdots \\
P_{\theta_{r e f}(k)}
\end{array}\right]^{+}\left[\begin{array}{c}
\mathbf{a}_{\theta_{r e f}(1)}^{m} \\
\vdots \\
\mathbf{a}_{\theta_{r e f}(k)}^{m}
\end{array}\right] .
$$

Detail analyses of reference view effects on gait identification performance is found in [20]. 

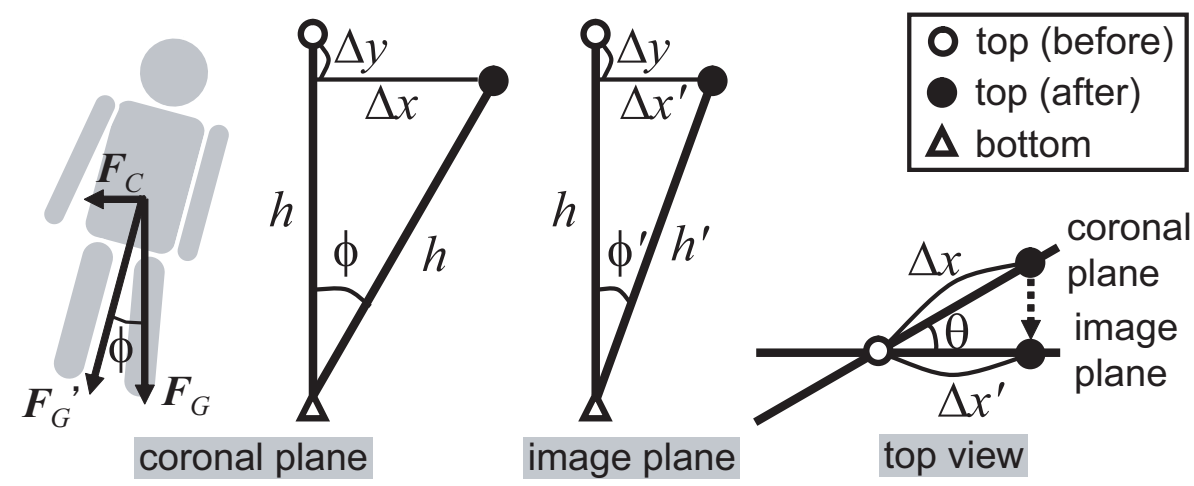

image plane

top view

Figure 3: Body tilt by centrifugal force

\section{Silhouette Correction by Body Tilt Estimation}

As described in section 1, when the person gradually changes his/her walking directions, he/she receives not only the gravitational force $\mathbf{F}_{G}=m \mathbf{g}$ but also the centrifugal force $\mathbf{F}_{C}$, where $m$ is the mass of the person and $\mathrm{g}$ is the gravitational acceleration. Thus, these forces sum up to the pseudo gravitational force $\mathbf{F}_{G}^{\prime}$ and make his/her body tilt into the same direction as $\mathbf{F}_{G}^{\prime}$ (see Fig. 3(a)) to keep his/her balance. When matching gait sequences with and without walking direction changes in the same condition, these body tilts should be corrected in advance. In this section, body tilt estimation is first addressed and subsequently silhouette correction based on the estimated body tilt is described.

\subsection{Body Tilt Estimation}

The first step is walking trajectory extraction. The position $\mathbf{X}=(Z, X)$ of the person in a camera coordinate (see Fig. 4) is obtained based on the object-to-image height ratio in the same way as [21],

$$
X=\frac{H}{h} x, Z=\frac{H}{h} f,
$$

where $H$ and $h$ are the heights of the person in the camera coordinate and in the image plane respectively, and $f$ is the focal length. We assume that $H$ is known for each person and that $f$ is obtained by camera calibration in advance. Moreover, the smoothing filter for temporal axis is applied to obtain a steady trajectory. An example of an estimated trajectory for a circular path is shown in Fig. 5.

Next step is centrifugal force estimation. Suppose that the walking trajectory consists of a set of arcs and that the person moves along the circular arc. Then, the centrifugal force $\mathbf{F}_{C}$ is

$$
\left|\mathbf{F}_{C}\right|=\frac{m|\mathbf{v}|^{2}}{r}
$$

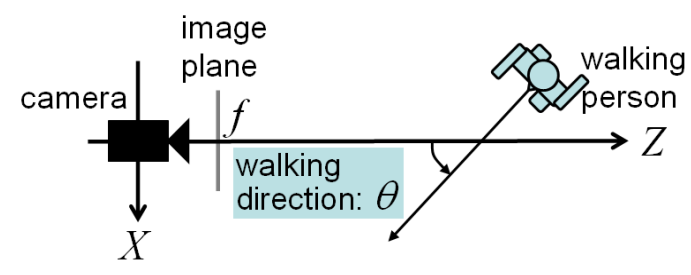

Figure 4: Definition of camera coordinate and walking direction $\theta$ at top view 


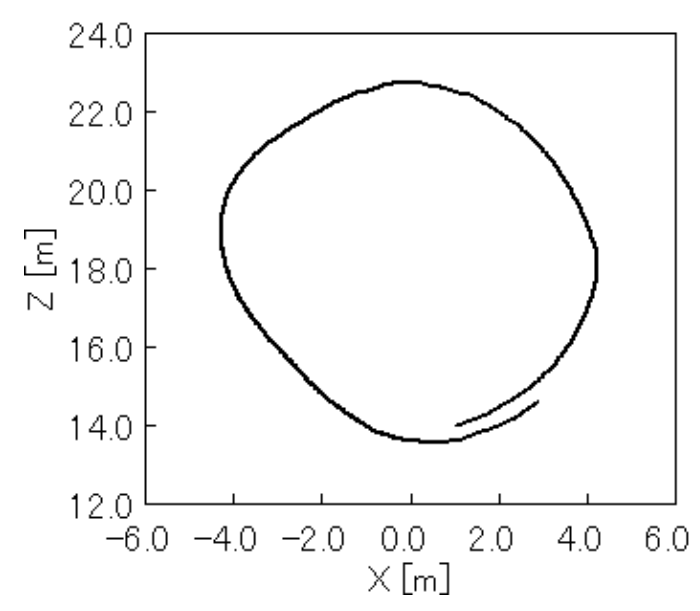

Figure 5: Example of estimated trajectory

where $\mathbf{v}$ is the walking velocity, and $r$ is curvature radius for the circular arc. Those for $n$th frame are simply calculated from central, forward, and backward differences of the walking trajectory as follows.

$$
\begin{aligned}
\mathbf{v}_{n} & =\frac{\mathbf{X}_{n+\Delta n}-\mathbf{X}_{n-\Delta n}}{2 \Delta n} \\
\mathbf{v}_{f n} & =\frac{\mathbf{X}_{n+\Delta n}-\mathbf{X}_{n}}{\Delta n} \\
\mathbf{v}_{b n} & =\frac{\mathbf{X}_{n}-\mathbf{X}_{n-\Delta n}}{\Delta n} \\
r_{n} & =\cos ^{-1}\left(\frac{\mathbf{v}_{f n}^{T} \mathbf{v}_{b n}}{\left|\mathbf{v}_{f n}\right|\left|\mathbf{v}_{b n}\right|}\right),
\end{aligned}
$$

where $\Delta n$ is frame step for calculating centeral, forward, and backward difference. The estimated walking speed and curvature radius for the circular path are shown in Fig. 6.

Finally, the body tilt angle $\phi$ is simply obtained based on force balance as

$$
\phi=\tan ^{-1}\left(\frac{\left|\mathbf{F}_{C}\right|}{\left|\mathbf{F}_{G}\right|}\right)=\tan ^{-1}\left(\frac{|\mathbf{v}|^{2}}{r|\mathbf{g}|}\right) .
$$

Figure 7 shows estimated walking direction $\theta$ and body tilt $\phi$ for the circular path in Fig. 5. Note that the walking direction $\theta$ is defined as Fig. 4 and easily obtained by the walking velocity $\mathbf{v}$.

\subsection{Silhouette Correction}

In this subsection, silhouette correction based on the estimated body tilt angle $\phi$ and walking direction $\theta$ is addressed. The effect of the body tilt angle $\phi$ on the gait silhouette is dependent on the walking direction $\theta$. Concretely speaking, if weak perspective projection is assumed, the body tilt is observed as rotation in image plane when walking direction $\theta$ is near 0 or $180 \mathrm{deg}$ (frontal view), and it is observed as vertical scaling in image plane when $\theta$ is near 90 or $270 \mathrm{deg}$ (side view). Here, we focus on the position shift of the top of head before and after body tilt when the foot point is fixed (see Fig. 3), and calculate the rotation angle and the vertical scale in the image plane to cancel the body tilt effects.

First, a horizontal and a vertical shift $\Delta x, \Delta y$ of the top of head in the coronal plane (see Fig. 3(a)) are

$$
\begin{aligned}
\Delta x & =h \sin \phi \\
\Delta y & =h(1-\cos \phi) .
\end{aligned}
$$




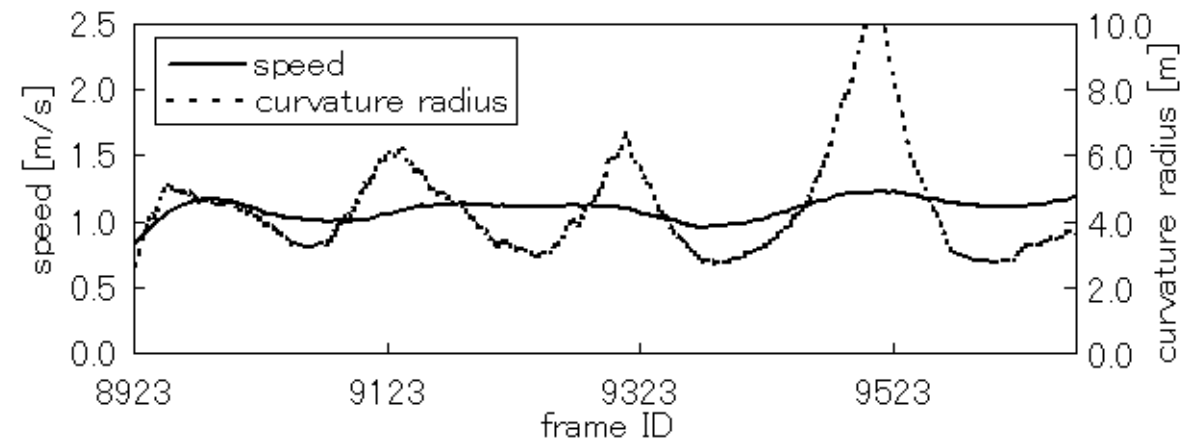

Figure 6: Estimated speed and curvature radius

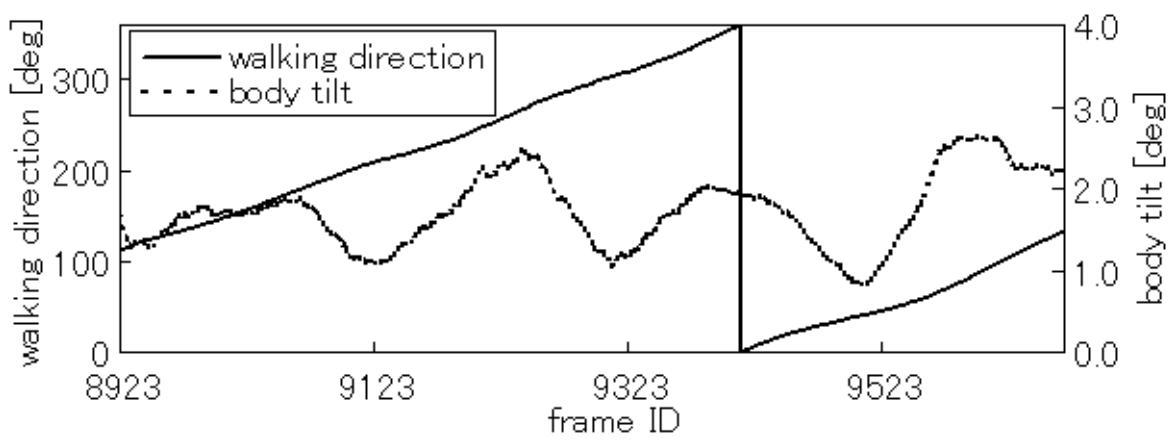

Figure 7: Estimated walking direction and body tilt

Next, a horizontal shift $\Delta x^{\prime}$ in the image plane (see Fig. 3 (b)) should be obtained. Considering the walking direction $\theta$ in the top view (see Fig. 3(c)), $\Delta x^{\prime}$ is

$$
\Delta x^{\prime}=\Delta x \cos \theta=h \sin \phi \cos \theta .
$$

Thus, rotation angle $\phi^{\prime}$ in the image plane is

$$
\phi^{\prime}=\tan ^{-1}\left(\frac{\Delta x^{\prime}}{h-\Delta y}\right)=\tan ^{-1}(\tan \phi \cos \theta) .
$$

Moreover, an apparent height $h^{\prime}$ in the image plane, that is, distance between foot point and the top of head after body tilt is

$$
h^{\prime}=\sqrt{\Delta x^{\prime 2}+(h-\Delta y)^{2}}=h \sqrt{\sin ^{2} \phi \cos ^{2} \theta+\cos ^{2} \phi} .
$$

Thus, the vertical scale $s_{y}$ is

$$
s_{y}=\frac{h^{\prime}}{h}=\sqrt{\sin ^{2} \phi \cos ^{2} \theta+\cos ^{2} \phi}
$$

Finally, a gait silhouette before body tilt is obtained by rotating the tilted silhouette by $\left(-\phi^{\prime}\right)$ and by scale it by $\left(1 / s_{y}\right)$ for vertical direction.

Figure 8 shows how rotation angle $\phi^{\prime}$ and vertical scale $s_{y}$ in the image plane change for each walking direction $\theta$ when the body tilt angle $\phi$ is fixed ( $2.0 \mathrm{deg}$ in this figure). We can see that rotation in the image plane is dominant near 0 and $180 \mathrm{deg}$ (front view) and that the vertical scaling is dominant near 90 and $270 \mathrm{deg}$ (side view). The vertical scale change is, however, at most $|1.0-\cos \phi|(=0.0006)$ and is negligible (regarded as $\cos \phi \simeq 1$ ) when the body tilt angle $\phi$ is relatively small. On the other hand, the rotation $\phi^{\prime}$ in the image plane is almost proportional to the body tilt $\phi$ in case of near front view and it cannot be negligible. 


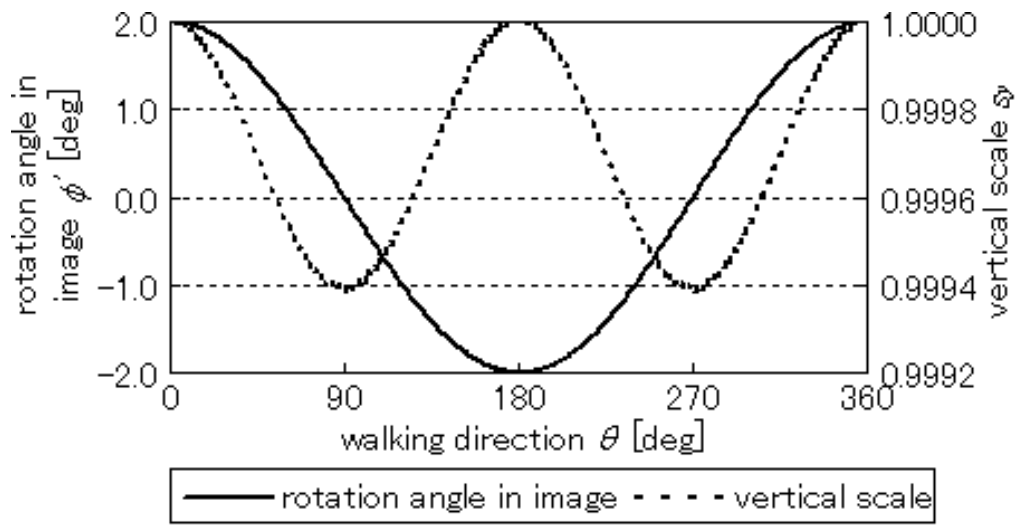

Figure 8: Changes of image rotation and vertical scale $(\phi=2.0)$

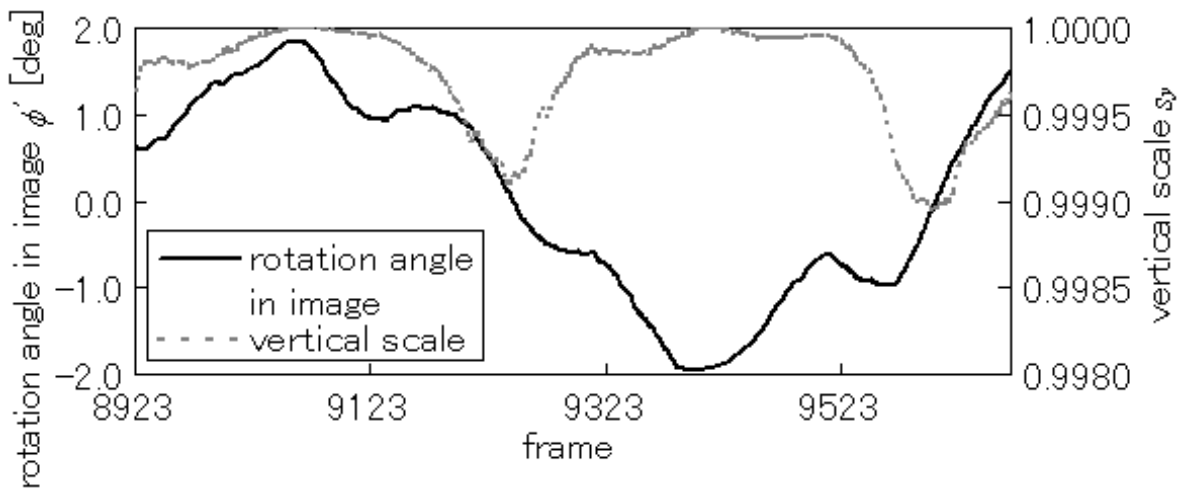

Figure 9: Estimated image rotation and vertical scale

Figure 9 shows rotation angle $\phi^{\prime}$ and vertical scale $s_{y}$ estimated from the circular trajectory in Fig. 5. Take correspondence to the estimated walking direction $\theta$ in Fig. 7 into consideration, we can see that the rotation angle $\phi^{\prime}$ in the image plane becomes large (approximately $\pm 2.0 \mathrm{deg}$ ) for frontal view ( 0 and $180 \mathrm{deg}$ ), and is approximately 0 for side view (90 and $270 \mathrm{deg}$ ). The vertical scale $s_{y}$ is 1.0 for frontal view and slightly decrease near the side view.

Figure 10 shows gait silhouettes before and after silhouette correction for the circular path in Fig. 5. Gait silhouettes near frontal view (from 2 nd to 5 th images) before correction are rotated counterclockwise by centrifugal force and they are almost canceled after the correction (The differences can be seen in the subtraction images). On the other hand, those near side view (9th and 10th images) are almost the same before/after correction and thus the differences cannot be seen in the subtraction images.

\section{Experiments}

In this section, experiments of gait identification for straight-walk and circular-walk sequences are described.

\subsection{Datasets}

For evaluating body tilt correction, the gait dataset should include curved-path sequences accompanied with camera calibration data. There are, however, no such gait database, thus we made our own gait dataset for evaluation. Moreover, because this paper focuses on gait identification itself, silhouette extraction is simplified by 

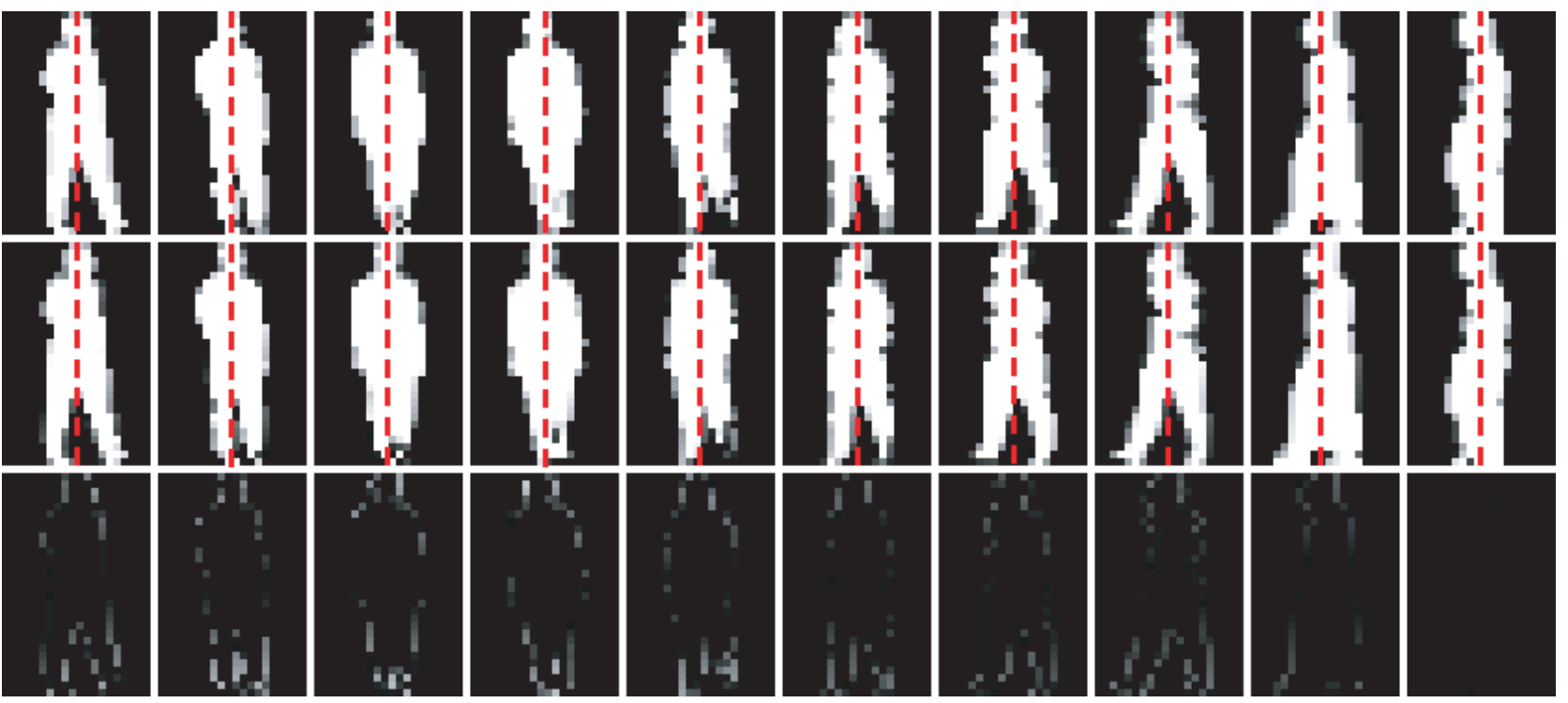

Figure 10: Results of gait silhouette correction (top: before correction, middle: after correction, bottom: subtraction images of before/after correction (every 30 frames))

exploiting temperature-based background subtraction using an infrared-ray camera, and by some assumptions that there are no camera motions and that camera setup is parallel to the ground.

We use a total of 744 straight-walk sequences from 20 subjects and 15 circular-walk sequences ( 3 rounds) from 15 subjects. The straight-walk sequences are quantized at every 15 directions, and then the number $K$ of directions is 24 . The training set for the VTM is composed of 480 straight-walk sequences of 20 subjects from 24 view directions. The gallery sets from 24 directions for each subject are constructed by transforming 3 references $(0,90$, and $180 \mathrm{deg})$ based on the trained VTM.

Probe sets (test sets) are composed of the other straight-walk sequences and the circular-walk sequences. For the circular-walk sequences, we extracted two types of features with and without body tilt correction. In the following subsections, we denote for convenience probe sets of the straight-walk sequence, and the circularwalk sequences with and without body tilt correction as "straight", "circular w/ BTC", and "circular w/o BTC", respectively.

\subsection{Results}

Performance is evaluated by a verification rate at $10 \%$ false positive rate in a Receiver Operating Characteristics (ROC) [22] curve. Figure 11 shows the verification rate when each subsequence including one gait period is matched separately for each walking direction. In this graph, directions of "straight" are limited for every 45 deg due to dataset limitation. We can see that body tilt correction improves the performance for circular-walk sequences as a whole. Although the performance improvements near side-view directions are not remarkable, those near frontal-view directions are outstanding (up to 30\%). This is because image rotation near frontalviews influences performance much more than vertical scaling near side-views as described in the previous section. In addition, the performance of circular-walk sequences after body tilt correction is competitive to that of straight-walk sequence. This fact shows the proposed method sufficiently corrects the body tilt by walking direction changes.

Next, Fig. 12 shows average verification rates when multiple periods of subsequences are used to matching. As a result, the performance improves as the number of periods increase, and the performance of circularwalk sequence with body tilt correction is always superior to that without body tilt correction. Moreover the performance of circular-walk sequence with body tilt correction is superior to that of straight-walk sequence 


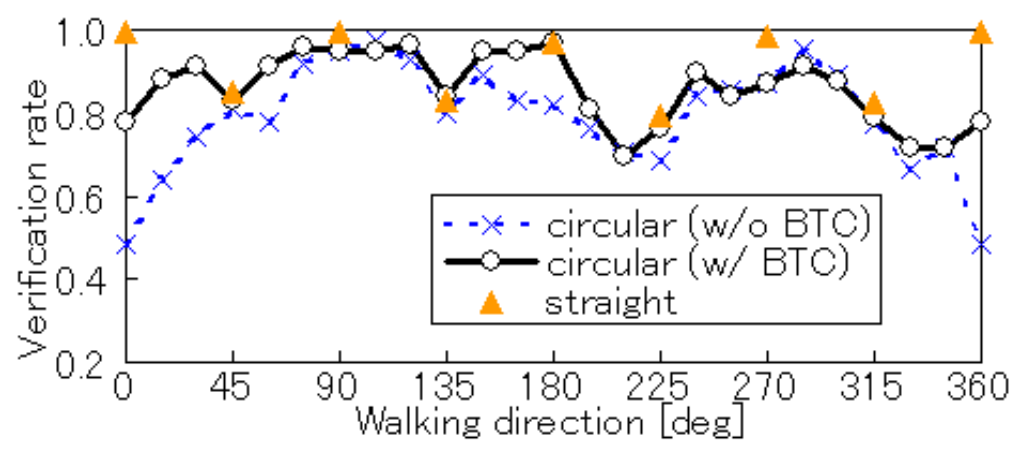

Figure 11: Verification rate for each walking direction

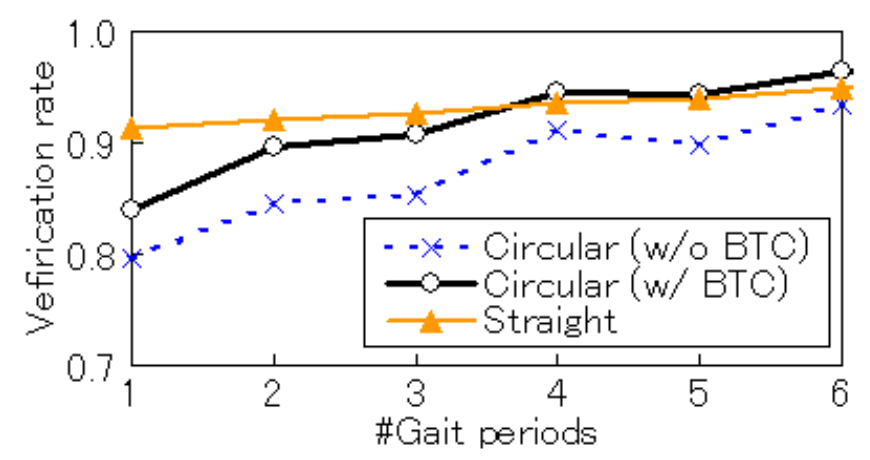

Figure 12: Verification rate for each number of gait periods

when the number of gait periods is more than 3. This result implies that view variations by walking direction changes are rather useful for gait identification when once the body tilts are corrected compared with straightwalk sequence.

\section{Conclusion and future works}

We have described a method of mechanics-based gait silhouette correction to cancel the body tilts by walking direction changes. The body tilt is model by a balance of gravitational force and centrifugal force induced by walking direction changes. A walking trajectory is approximated by a set of arcs piecewise and the centrifugal force is estimated by circular motion assumption along the arcs. Then, the silhouette is rotated and vertically scaled to cancel the body tilt effects in the image plane based on the estimated body tilt angle and the walking direction.

Experiments of gait identification for straight-walk and circular-walk sequences were made to confirm the effectiveness of the proposed body tilt correction. As a result, performance improvement up to $30 \%$ verification rate (at 10\% false alarm rate) was observed for near frontal-view directions where the image rotation correction was dominant. Moreover, the number of gait periods for circular-walk sequences turned out to have more influence on identification performance than that for straight-walk sequences. This seems to be derived from view variations provided by walking direction changes from circular-walk sequences and implies the possibility of performance improvement by multi-view gait observations. Such view variations are generated not only walking direction changes but also view direction changes from a camera. Han et al. [23] used view direction changes for a straight-walk sequence to reduce a view difference between a probe and a gallery. The view variation was, however, so small to generate sufficient view variations. Therefore, multi-view gait identification 
utilizing view variations provided by wide-view cameras is one of future research directions. Other future works are adaptation to more generic view-point changes including camera tilts and experiments for a general database such as the HumanID Gait Challenge Problem Datasets [8]. Particularly, experimental dateset in this paper was composed of relatively clear silhouette sequences thanks to temperature-based background subtraction using a infrared-ray camera, therefore experimental validation using noisy silhouette sequences due to imperfect background subtraction and occlusion is necessary.

\section{References}

[1] M. Blank, L. Gorelick, E. Shechtman, M. Irani, and R. Basri: Actions as space-time shapes. In Proc. of 10th Int. Conf. on Computer Vision 2. 1395-1402 2005

[2] M. Vasilescu: Human motion signatures: analysis, synthesis, recognition. In Proc. of the Int. Conf. on Pattern Recognition 3. 456-460 2002

[3] M. Nixon and J. Carter: Automatic recognition by gait. Proc. of the IEEE 94(11): 2013-2024 2006

[4] C. Yam, M. Nixon, and J. Carter: Automated person recognition by walking and running via model-based approaches. Pattern Recognition 37(5): 1057-1072 2004

[5] R. Urtasun and P. Fua: 3d tracking for gait characterization and recognition. In Proc. of the 6th IEEE Int. Conf. on Automatic Face and Gesture Recognition. 17-22 2004

[6] R. Tanawongsuwan and A. Bobick: Modelling the effects of walking speed on appearance-based gait recognition. In Proc. of IEEE Conf. on Computer Vision and Pattern Recognition 2. 783-790 2004

[7] A. Bobick and A. Johnson: Gait recognition using static activity-specific parameters. In Proc. of IEEE Conf. on Computer Vision and Pattern Recognition 1. 423-430 2001

[8] S. Sarkar, J. Phillips, Z. Liu, I. Vega, P. Grother, and K. Bowyer: The humanid gait challenge problem: Data sets, performance, and analysis. Trans. of Pattern Analysis and Machine Intelligence 27(2): 162-177 2005

[9] J. Han and B. Bhanu: Individual recognition using gait energy image. Trans. on Pattern Analysis and Machine Intelligence 28(2): 316- 3222006

[10] T. Kobayashi and N. Otsu: Action and simultaneous multiple-person identification using cubic higherorder local auto-correlation. In Proc. of the 17th Int. Conf. on Pattern Recognition 3. 741-744 2004

[11] R. Sagawa, Y. Makihara, T. Echigo, and Y. Yagi: Matching gait image sequences in the frequency domain for tracking people at a distance. In Proc. of the 7th Asian Conf. on Computer Vision 2. 141-150 2006

[12] S. Yu, D. Tan, and T. Tan: Modelling the effect of view angle variation on appearance-based gait recognition. In Proc. of 7th Asian Conf. on Computer Vision 1. 807-816 2006

[13] D. Weinland, R. Ronfard, and E. Boyer: Automatic discovery of action taxonomies from multiple views. In Proc. of the 2006 IEEE Computer Society Conf. on Computer Vision and Pattern Recognition 2., New York, USA 1639-1645 2006

[14] G. Shakhnarovich, L. Lee, and T. Darrell: Integrated face and gait recognition from multiple views. In Proc. of IEEE Conf. on Computer Vision and Pattern Recognition 1. 439-446 2001

[15] A. Kale, A. Roy-Chowdhury, and R. Chellappa: Towards a view invariant gait recognition algorithm. In Proc. of IEEE Conf. on Advanced Video and Signal Based Surveillance. 143-150 2003 
[16] Y. Makihara, R. Sagawa, Y. Mukaigawa, T. Echigo, and Y. Yagi: Gait recognition using a view transformation model in the frequency domain. In Proc. of the 9th European Conf. on Computer Vision 3., Graz, Austria 151-1632006

[17] A. Utsumi and N. Tetsutani: Adaptation of appearance model for human tracking using geometrical pixel value distributions. In Proc. of the 6th Asian Conf. on Computer Vision 2. 794-799 2004

[18] C.-S. Lee and A. Elgammal: Gait style and gait content: bilinear models for gait recognition using gait re-sampling. In Proc. of 6th IEEE Conf. on Automatic Face and Gesture Recognition. 147-152 2004

[19] F. Cuzzolin: Using bilinear models for view-invariant action and identity recognition. In Proc. of the 2006 IEEE Computer Society Conf. on Computer Vision and Pattern Recognition 2., New York, USA $1701-17082006$

[20] Y. Makihara, R. Sagawa, Y. Mukaigawa, T. Echigo, and Y. Yagi: Which reference view is effective for gait identification using a view transformation model? In Proc. of the IEEE Computer Society Workshop on Biometrics 2006, New York, USA 2006

[21] M. Hild: Estimation of 3d motion trajectory and velocity from monocular image sequences in the context of human gait recognition. In Proc. of the 17th Int. Conf. on Pattern Recognition 1. 231-235 2004

[22] P. Phillips, H. Moon, S. Rizvi, and P. Rauss: The feret evaluation methodology for face-recognition algorithms. Trans. of Pattern Analysis and Machine Intelligence 22(10): 1090-1104 2000

[23] J. Han, B. Bhanu, and A. Roy-Chowdhury: A study on view-insensitive gait recognition. In Proc. of IEEE Int. Conf. on Image Processing 3. 297-300 2005 